\title{
Ultra-compact broadband higher order-mode pass filter fabricated in a silicon waveguide for multimode photonics
}

\author{
Guan, Xiaowei; Ding, Yunhong; Frandsen, Lars Hagedorn
}

Published in:

Optics Letters

Link to article, DOI:

$10.1364 /$ ol. 40.003893

Publication date:

2015

Document Version

Publisher's PDF, also known as Version of record

Link back to DTU Orbit

Citation (APA):

Guan, X., Ding, Y., \& Frandsen, L. H. (2015). Ultra-compact broadband higher order-mode pass filter fabricated in a silicon waveguide for multimode photonics. Optics Letters, 40(16), 3893-3896.

https://doi.org/10.1364/ol.40.003893

\section{General rights}

Copyright and moral rights for the publications made accessible in the public portal are retained by the authors and/or other copyright owners and it is a condition of accessing publications that users recognise and abide by the legal requirements associated with these rights.

- Users may download and print one copy of any publication from the public portal for the purpose of private study or research.

- You may not further distribute the material or use it for any profit-making activity or commercial gain

- You may freely distribute the URL identifying the publication in the public portal 


\title{
Ultra-compact broadband higher order-mode pass filter fabricated in a silicon waveguide for multimode photonics
}

\author{
Xiaowe Guan, ${ }^{*}$ Yunhong Ding, and Lars H. Frandsen \\ Department of Photonics Engineering, Technical University of Denmark, Orsteds Plads Building 345A, 2800 Kgs. Lyngby, Denmark \\ ${ }^{*}$ Corresponding author: xgua@fotonik.dtu.dk
}

Received 8 July 2015; accepted 19 July 2015; posted 29 July 2015 (Doc. ID 245566); published 13 August 2015

An ultra-compact and broadband higher order-mode pass filter in a $1 \mathrm{D}$ photonic crystal silicon waveguide is proposed and experimentally demonstrated. The photonic crystal is designed for the lower order mode to work in the photonic band gap, while the higher order mode is located in the air band. Consequently, light on the lower order mode is prohibited to pass through the filter, while light on a higher order mode can be converted to a Bloch mode in the photonic crystal and pass through the filter with low insertion loss. As an example, we fabricate a $\sim 15-\mu \mathrm{m}$-long first-order-mode pass filter that filters out the fundamental mode and provides a measured insertion loss of $\sim 1.8 \mathrm{~dB}$ for the first-order-mode pass signals. The extinction ratio is measured to be around $50 \mathrm{~dB}$ (with a variation of $\pm 10 \mathrm{~dB}$ due to the detection limitation of the measurement setup) in the measured wavelength range from 1480 to $1580 \mathrm{~nm}$. Additionally, calculations predict the extinction ratio to be larger than $50 \mathrm{~dB}$ in a $170 \mathrm{~nm}$ broad bandwidth. (๑) 2015 Optical Society of America

OCIS codes: (130.3120) Integrated optics devices; (030.4070) Modes; (130.5296) Photonic crystal waveguides; (230.7390) Waveguides, planar.

http://dx.doi.org/10.1364/OL.40.003893

In order to keep up with the ever-increasing demands for higher capacity in optical communication systems, transmitting signals with multiple modes in a few-mode fiber (FMF) has recently been suggested as a promising technique [1-3]. Being an integrated version of this technique, multimode photonics in silicon waveguides has attracted substantial attention [4-7] to exploit the already matured single-mode silicon photonic devices and silicon's complementary metal-oxidesemiconductor (CMOS) compatibility. For devices applied in the silicon multimode photonics, various functionalities have been achieved including (de)multiplexing [8-11], bending [12], converting [13], and resonating [14] multimode signals. Additionally, mode filtering is also foreseen as an essential functionality in silicon multimode photonics for mode-division multiplexing (MDM), resembling wavelength filtering for wavelength-division multiplexing (WDM). Filtering out a higher order mode in a waveguide is not a tough problem due to its weaker confinement. Simple solutions can be implemented, e.g., tapering the waveguide down to the cut-off width of the higher order mode or stripping the higher order mode in an appropriately designed waveguide bend [15]. In both cases, signals carried on the higher order mode are naturally lost. However, a higher order-mode pass filter, which can keep a signal on a higher order mode but filter out light on the lower order mode, is not straightforward to produce. Meanwhile, for on-chip applications, mode filters are supposed to have a compact size without compromising insertion loss (IL) and extinction ratio (ER). To the best of our knowledge, there have been no reports in literature on ultra-compact higher ordermode pass filters. Although an add/drop configuration can be seen as a higher order-mode pass filter, it still encounters the drawback of having a large footprint (e.g., $>40 \mu \mathrm{m}$ for adding and dropping the transverse electric first-order-mode [16]).

In this Letter, we propose and experimentally demonstrate an ultra-compact higher order-mode (HOM) pass filter using a $1 \mathrm{D}$ photonic crystal $(\mathrm{PhC})$ in a silicon $(\mathrm{Si})$ multimode waveguide. The $1 \mathrm{D}$ PhC has previously been used in a silicon singlemode waveguide to accomplish a 9- $\mu \mathrm{m}$-long polarization filter with an ER of $27 \mathrm{~dB}$ [17]. Here, the $1 \mathrm{D} \mathrm{PhC}$ is applied in a silicon multimode waveguide to achieve a $\mathrm{HOM}$ pass filter, and the ER is measured to be around $50 \mathrm{~dB}$ with a filter length $\sim 15 \mu \mathrm{m}$.

Figure 1 shows the 3D view of the proposed HOM pass filter with a $1 \mathrm{D} \mathrm{PhC}$ in a silicon waveguide. The $\mathrm{PhC}$ is comprised of a periodic corrugation of the silicon waveguide with period $L_{p}$ and width $w_{b}$ of the connecting nanobridge. To reduce the insertion loss originating from the mode mismatch for the $\mathrm{HOM}$, adiabatic tapers are designed to access the $\mathrm{PhC}$ with the silicon waveguides, the width of which, $w_{\mathrm{ac}}$, is larger than $500 \mathrm{~nm}$ to support multimodes as shown by the cross-section of the inset in Fig. 1.

It is well known that a higher order mode in a silicon wire has a weaker confinement than a lower order mode (e.g., as indicated by the mode profiles of the $\mathrm{TE}_{0}$ and the $\mathrm{TE}_{1}$ modes in Fig. 2) and, therefore, the higher order mode has a lower 


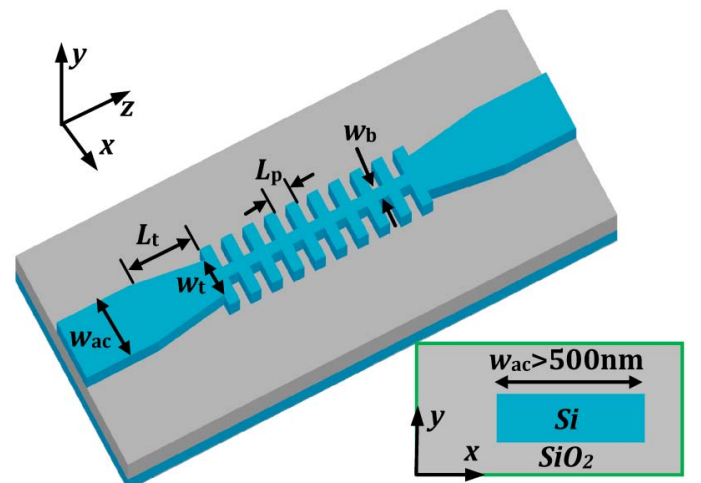

Fig. 1. 3D view of the proposed higher order-mode pass filter. Inset shows the cross-section of the access multimode waveguides with width $w_{\mathrm{ac}}>500 \mathrm{~nm}$.

effective index. Thus, in order to realize the HOM pass filter, the $\mathrm{PhC}$ should be designed for the lower order mode to be located in the band gap while the higher order mode should lie in the air band of the PhC. Consequently, the lower order mode is reflected and/or scattered while the higher order mode will be converted to a Bloch mode in the $\mathrm{PhC}$ and propagate through the filter with low insertion loss. Since the PhC is based on a periodic grating structure, one can estimate the structural parameters by approximately satisfying the following conditions:

$$
\begin{aligned}
& \left(n_{b}^{\text {lower }}+n_{t}^{\text {lower }}\right) L_{p} / 2=\lambda_{0} / 2, \\
& \left(n_{b}^{\text {higher }}+n_{t}^{\text {higher }}\right) L_{p} / 2<\lambda_{0} / 2,
\end{aligned}
$$

where $\lambda_{0}$ is the central wavelength, $L_{p}$ is the period (filling factor 0.5 ), and $n_{b}$ and $n_{t}$ are the effective indices of the lower or higher order modes in the nanobridge and teeth of the grating, respectively. Not only should the effective indices of the lower order mode in the designed filter satisfy the Bragg grating equation in Eq. (1), but the optical path length for the higher order mode should also be quite smaller than half the central wavelength in order to be far away from the cut-off condition, which is expressed by Eq. (2).

As a design example, we choose the transverse-electric (TE) fundamental mode $\left(\mathrm{TE}_{0}\right)$ and the first-order mode $\left(\mathrm{TE}_{1}\right)$ as the lower and higher order modes, respectively, to realize a

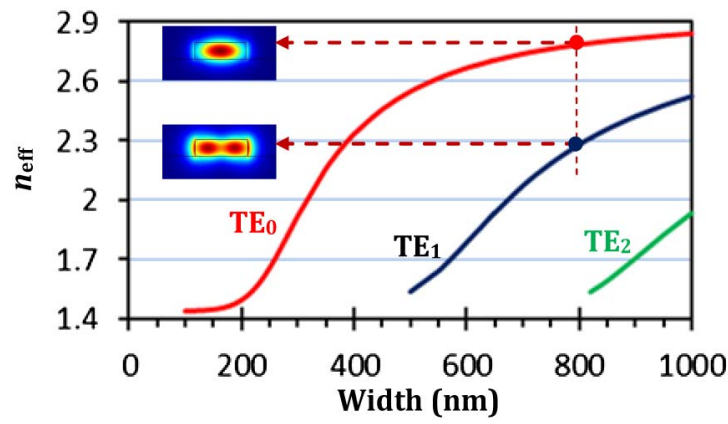

Fig. 2. Effective indices for the TE eigenmodes calculated for different widths of a 250 -nm-thick silicon waveguide. Here the wavelength is $1550 \mathrm{~nm}$. Insets show the mode profiles of the $\mathrm{TE}_{0}$ and the $\mathrm{TE}_{1}$ modes for a waveguide width of $800 \mathrm{~nm}$.
HOM $\mathrm{TE}_{1}$ pass filter. Figure 2 shows the effective indices $n_{\text {eff }}$ calculated using the finite-element method implemented in the software package COMSOL for different TE modes and different widths of the silicon waveguide having a height of $250 \mathrm{~nm}$. Here, the wavelength $\lambda_{0}=1550 \mathrm{~nm}$, and the refractive indices of $\mathrm{Si} n_{\mathrm{Si}}=3.478$ and the surrounding silica $\left(\mathrm{SiO}_{2}\right) \quad n_{\mathrm{SiO} 2}=1.444$ are calculated from the Sellmeier formula [18]. By choosing the width of the access waveguides $w_{\mathrm{ac}}=800 \mathrm{~nm}$ and the width of the nanobridge $w_{b}=$ $140 \mathrm{~nm}$, we obtain a grating period of $\sim 410 \mathrm{~nm}$, in which the optical path length of the $\mathrm{TE}_{1}$ mode is $\sim 0.636 \mu \mathrm{m}$ and smaller than half a wavelength $(0.775 \mu \mathrm{m})$. Here, $n_{b}$ of the higher order $\left(\mathrm{TE}_{1}\right)$ mode used in Eq. (2) is approximately 1.444 as the $\mathrm{TE}_{1}$ mode is not supported in the nanobridge with a width of $140 \mathrm{~nm}$ and therefore will be distributed in the surrounding $\mathrm{SiO}_{2}$.

Using a 3D finite-difference time-domain (3D FDTD) method, the band diagram of the $\mathrm{PhC}$ can be obtained, and the grating period $L_{p}$ is optimized to be $370 \mathrm{~nm}$ to center $1550 \mathrm{~nm}$ in the band gap for the $\mathrm{TE}_{0}$ mode, as shown in Fig. 3. At the wavelength of $1550 \mathrm{~nm}$, the wavevector $(k)$ of the $\mathrm{TE}_{1}$ Bloch mode is $6.928 \mu \mathrm{m}^{-1}$, and the corresponding effective index $\left(n_{\text {eff }}\right)$ is 1.709 according to the equation $k=(2 \pi / \lambda) n_{\text {eff. }}$. From this value and Fig. 2 , we choose a waveguide width of the taper $\left(w_{t}\right)$ of $575 \mathrm{~nm}$ to mode (index)-match the $\mathrm{TE}_{1}$ waveguide mode in the access waveguide to the $\mathrm{TE}_{1}$ Bloch mode in the PhC.

Figure 4(a) shows the calculated normalized transmissions for the $\mathrm{TE}_{0}$ (red) and $\mathrm{TE}_{1}$ (blue) modes in the designed $\mathrm{TE}_{1}$-mode pass filter with a period number $N=20$. Here, the taper length $L_{t}$ is $4 \mu \mathrm{m}$, i.e., the total length of the filter is $\sim 15 \mu \mathrm{m}$. One can find that the transmission for the $\mathrm{TE}_{0}$ mode is very low $(<-50 \mathrm{~dB}$ ) from $1450 \mathrm{~nm}$ to $1620 \mathrm{~nm}$. In contrast, the transmission for the $\mathrm{TE}_{1}$ mode has very low loss $(<3 \mathrm{~dB})$ in the same wavelength range. Specifically, the transmissions at $\lambda_{0}=1550 \mathrm{~nm}$ are $-60.7 \mathrm{~dB}$ and $-1.3 \mathrm{~dB}$ for the $\mathrm{TE}_{0}$ and $\mathrm{TE}_{1}$ modes, respectively. Figures $4(\mathrm{~b})$ and $4(\mathrm{c})$ show the simulated electrical fields of light propagation at the central wavelength $\lambda_{0}=1550 \mathrm{~nm}$ for the $\mathrm{TE}_{0}$ and the $\mathrm{TE}_{1}$ modes, respectively, and clearly picture the pass filter functionality.

The designed $\mathrm{TE}_{1}$-mode pass filters were fabricated on a silicon-on-insulator wafer with $250-\mathrm{nm}$ silicon on top of a

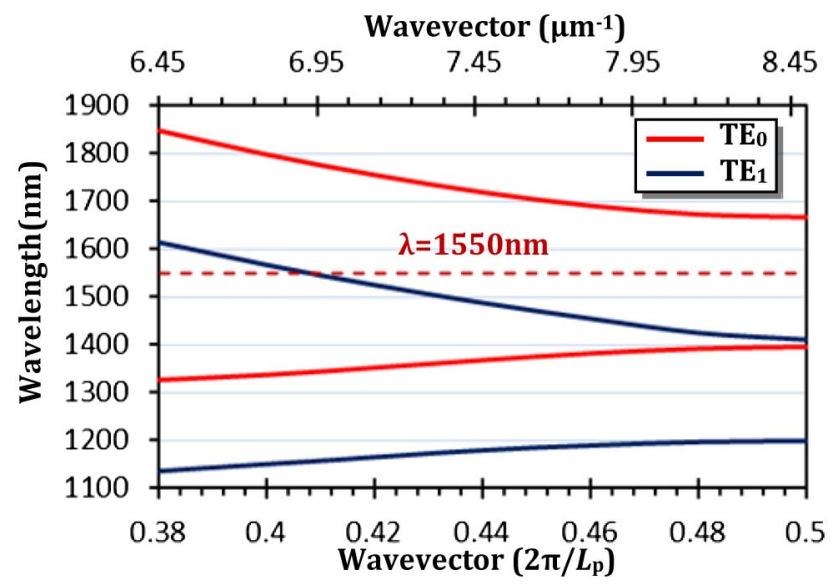

Fig. 3. Calculated band diagram of the grating-type 1D PhC. Here the period $L_{p}$ is $370 \mathrm{~nm}$. 

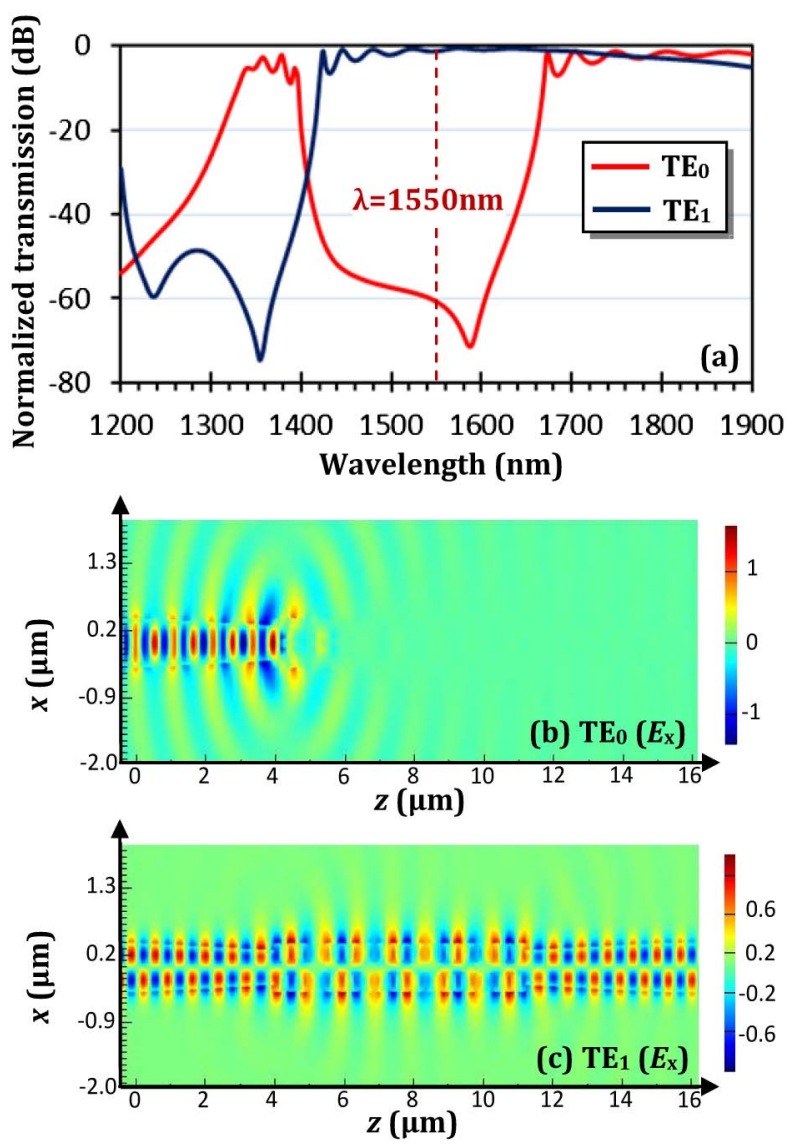

Fig. 4. Calculated normalized transmissions (a), and light propagation for the $\mathrm{TE}_{0}$ (b) and the $\mathrm{TE}_{1}$ (c) modes of the designed filter with $N=20\left(\lambda_{0}=1550 \mathrm{~nm}\right)$.

$3-\mu \mathrm{m}$ buried-oxide (BOX) layer. Fully etched grating couplers [19] were used to couple light between the fibers and the silicon waveguides. Electron-beam lithography was utilized to pattern the chip and followed by an inductive plasma-etching process [13]. Finally, the whole chip was covered with a $1-\mu \mathrm{m} \mathrm{SiO}_{2}$ layer. Figure 5 (bottom) shows the scanning electron micrograph (SEM) image of the fabricated filter with period number $N=20$, and Fig. 5 (top) shows a microscope image of the waveguide configuration for characterizing the pass filter. In the waveguide configuration, adiabatic tapers are used to couple the $\mathrm{TE}_{0}$ mode from a single-mode waveguide with a width of
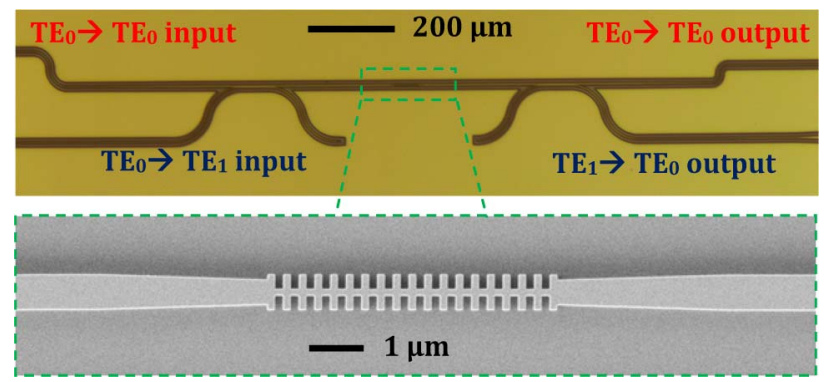

Fig. 5. (Top) Microscope image of the waveguide configuration for characterizing the proposed filter. (Bottom) SEM image of the fabricated HOM pass filter.
$450 \mathrm{~nm}$ to the $\mathrm{TE}_{0}$ mode in the access multimode waveguides having a width of $800 \mathrm{~nm}$. Meanwhile, asymmetrical directional couplers similar as in the Ref. [20] are used to add/drop the $\mathrm{TE}_{1}$ mode in the access multimode waveguides from/to the $\mathrm{TE}_{0}$ mode in the single-mode silicon waveguides.

In order to efficiently couple between the $\mathrm{TE}_{1}$ mode in the silicon multimode waveguide and the $\mathrm{TE}_{0}$ mode in the silicon single-mode waveguide, phase matching should be satisfied, i.e., the effective indices should be equal for the two modes. From Fig. 2, one can find that a width of $383 \mathrm{~nm}$ for the single-mode waveguide would secure phase matching to the multimode waveguide with the effective indices being $\sim 2.28$. Figure 6(a) shows the measured coupling efficiency for the twowaveguide-coupling system with a coupling length of $15 \mu \mathrm{m}$ and a gap of $200 \mathrm{~nm}$ as shown by the inset SEM image. Taking into account the measurement inaccuracy of $\sim 0.2 \mathrm{~dB}$, the coupling efficiency is $-0.3 \mathrm{~dB}(\sim 93 \%)$ at $1550 \mathrm{~nm}$ and larger than $-2 \mathrm{~dB}(\sim 63 \%)$ from $1490 \mathrm{~nm}$ to $1580 \mathrm{~nm}$. With this waveguides configuration, we measured the normalized transmissions of the $\mathrm{TE}_{0}$ and the $\mathrm{TE}_{1}$ modes for the proposed filter with period number $N=20$ as shown in Fig. 6(b), in which the calculated transmissions from Fig. 4(a) in the measured wavelength range are also given for comparison. The waveguides used for normalization have the same configuration but without the filter. One can see that for the whole measured wavelength range from 1480 to $1580 \mathrm{~nm}$, the measured transmission of the $\mathrm{TE}_{0}$ mode is as low as $-50 \mathrm{~dB}$. The
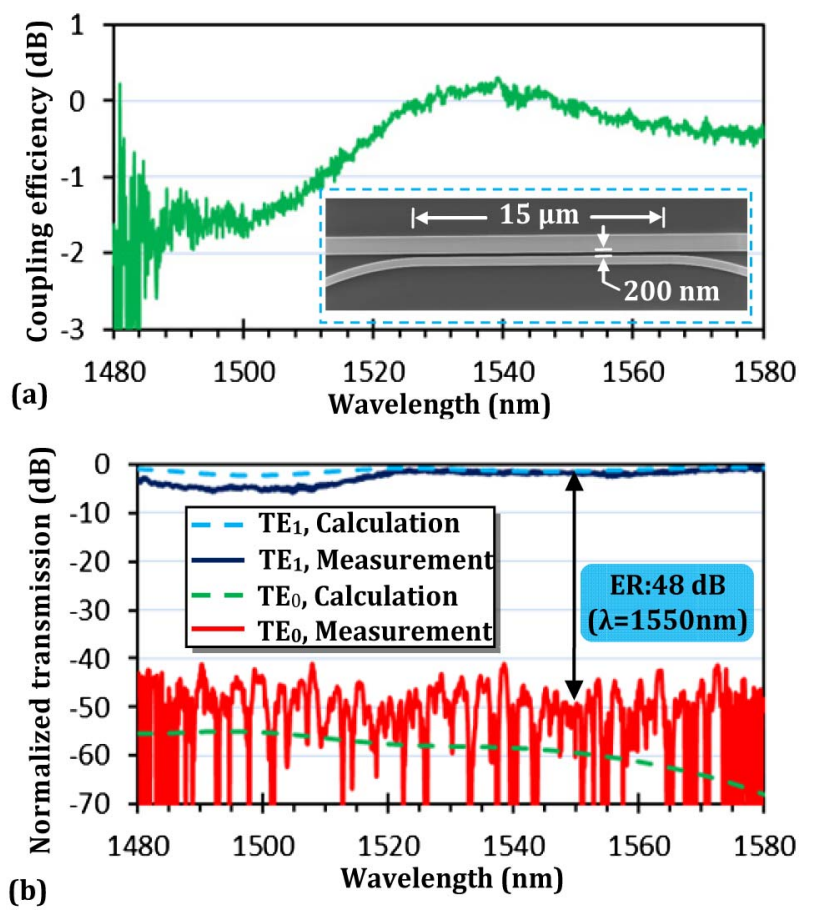

Fig. 6. (a) Measured coupling efficiency from $\mathrm{TE}_{0}$ mode in a waveguide with width $383 \mathrm{~nm}$ to the $\mathrm{TE}_{1}$ mode in a waveguide with width $800 \mathrm{~nm}$. Inset shows the SEM image of the coupled waveguides. Here, due to a measurement inaccuracy of $\sim 0.2 \mathrm{~dB}$, the measured and normalized coupling efficiency is slightly larger than $0 \mathrm{~dB}$ when it is close to full coupling around $1540 \mathrm{~nm}$. (b) Measured and normalized transmissions (solid lines) of the $\mathrm{TE}_{0}$ and the $\mathrm{TE}_{1}$ modes for the proposed filter with $N=20$. Here the calculated transmission spectra (dashed lines) from Fig. 4(a) are also given for comparison. 


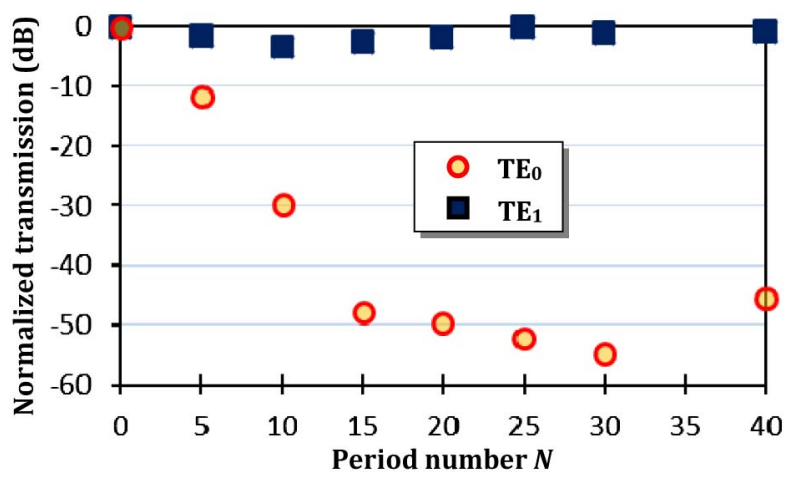

Fig. 7. Measured and normalized transmissions of the $\mathrm{TE}_{0}$ and $\mathrm{TE}_{1}$ modes for the filter as a function of period number $N$. Here the operating wavelength is $1550 \mathrm{~nm}$. The normalized waveguides have the same configuration but without the filter, i.e., $N=0$.

variation of $\pm 10 \mathrm{~dB}$ is due to the absolute measured power $(\sim-80 \mathrm{dBm})$ being close to the noise floor of our optical spectrum analyzer. The measured transmission level of the $\mathrm{TE}_{1}$ mode is larger than $-5 \mathrm{~dB}$ for the whole measured wavelength range. Thus, ERs of $\sim 50 \mathrm{~dB}$ can be achieved in the 100-nm band. At $1550 \mathrm{~nm}$, the ER for the fabricated $\mathrm{TE}_{1}$-mode pass filter is $48 \mathrm{~dB}$, and the IL is $1.8 \mathrm{~dB}$. From the figure, it is also seen that we obtain good agreement between measured and calculated values.

We also measured the normalized transmissions of the $\mathrm{TE}_{0}$ and the $\mathrm{TE}_{1}$ modes at $1550 \mathrm{~nm}$ for the proposed $\mathrm{TE}_{1}$-mode pass filter with different $\mathrm{PhC}$ period number $N$, as shown in Fig. 7. For all periods, one can find that the transmission of the $\mathrm{TE}_{1}$ mode is always larger than $-3.5 \mathrm{~dB}$. On the other hand, the transmission of the $\mathrm{TE}_{0}$ mode initially decreases linearly and starts to fluctuate when the transmission reaches below $-50 \mathrm{~dB}(N>15)$, i.e., being close to the detection limit of the setup. Therefore, one can reasonably expect a higher ER for the proposed filter using a setup with higher sensitivity.

In summary, we have proposed and demonstrated an ultracompact and broadband higher order-mode pass filter based on a $1 \mathrm{D} \mathrm{PhC}$ and fabricated in a silicon waveguide. The $1 \mathrm{D} \mathrm{PhC}$ is designed for the lower order mode to work in the band gap so that light on the lower order mode will be prohibited entering the $\mathrm{PhC}$ with high reflection and scattering. In contrast, the $\mathrm{PhC}$ supports a Bloch mode for the higher order mode so that light on the higher order mode can propagate through the filter with low loss. As an example, we designed and fabricated a $\mathrm{TE}_{1}$-mode pass filter rejecting the $\mathrm{TE}_{0}$ mode. The fabricated $\mathrm{TE}_{1}$-mode pass filter has an ER of $\sim 48 \mathrm{~dB}$ and an IL of $\sim 1.8 \mathrm{~dB}$ at $1550 \mathrm{~nm}$ when the period number of the $\mathrm{PhC}$ grating is 20 . The corresponding length of the filter is only $15 \mu \mathrm{m}$, including tapers and $\mathrm{a} \sim 7 \mu \mathrm{m}$-long $\mathrm{PhC}$ part. For the complete measured wavelength range of $100 \mathrm{~nm}$, ERs of $\sim 50 \mathrm{~dB}$ are achieved, which are limited by the detection limitation of our measurement setup. We believe one can cascade such filters to get a higher order-mode pass filter of any order. For example, a $\mathrm{TE}_{2}$-mode pass filter can be realized by cascading one filter filtering out the $\mathrm{TE}_{0}$ mode (keeping the $\mathrm{TE}_{2}$ and the $\mathrm{TE}_{1}$ modes) and one pass-filter filtering out the $\mathrm{TE}_{1}$ mode (keeping the $\mathrm{TE}_{2}$ mode). Thus, the present design provides a promising option to achieve an on-chip higher order-mode pass filter for higher order modes with any order and simultaneously having ultrahigh extinction ratios and broad band operation.

Funding. Det Frie Forskningsråd (Danish Council for Independent Research) (DFF-1335-00771, DFF-133700152); Villum Fonden (Villum Foundation) (VKR023112).

\section{REFERENCES}

1. R. Ryf, S. Randel, A. H. Gnauck, C. Bolle, A. Sierra, S. Mumtaz, M. Esmaeelpour, E. C. Burrows, R.-J. Essiambre, P. J. Winzer, D. W. Peckham, A. H. Mcurdy, and R. Lingle, J. Lightwave Technol. 30, 521 (2012).

2. S. Randel, R. Ryf, A. Sierra, P. J. Winzer, A. H. Gnauck, C. A. Bolle, R.-J. Essiambre, D. W. Peckham, A. McCurdy, and R. Lingle, Opt. Express 19, 16697 (2011).

3. M. Salsi, C. Koebele, D. Sperti, P. Tran, P. Brindel, H. Mardoyan, S. Bigo, A. Boutin, F. Verluise, P. Sillard, M. Astruc, L. Provost, F. Cerou, and G. Charlet, "Transmission at $2 \times 100-\mathrm{gb} / \mathrm{s}$ over two modes of $40 \mathrm{~km}$-long prototype few-mode fiber, using lcos based mode multiplexer and demultiplexer," in Optical Fiber Conference, Los Angeles, California, USA, (2011), paper PDPB9.

4. M. Greenberg and M. Orenstein, Opt. Express 13, 9381 (2005).

5. S. Bagheri and W. M. J. Green, "Silicon-on-insulator mode-selective add-drop unit for on-chip modedivision multiplexing," in Proceedings of IEEE Group IV Photonics Conference, San Francisco, California, USA, (2009), pp. 166-168.

6. L. H. Gabrielli, D. Liu, S. G. Johnson, and M. Lipson, "On-chip multimode photonics," in Quantum Electronics and Laser Science Conference, San Jose, California, USA, (2012), paper QTh5B. 4.

7. D. Dai, "Silicon mode-(de)multiplexer for a hybrid multiplexing system to achieve ultrahigh capacity photonic networks-on-chip with a singlewavelengthcarrier light," in Asia Communications and Photonics Conference, Guangzhou, China, (2012), paper ATh3B.3.

8. L.-W. Luo, N. Ophir, C. P. Chen, L. H. Gabrielli, C. B. Poitras, K. Bergmen, and M. Lipson, Nat. Commun. 5, 3069 (2014).

9. J. Wang, S. He, and D. Dai, Laser Photon. Rev. 8, L18 (2014).

10. J. B. Driscoll, R. R. Grote, B. Souhan, J. I. Dadap, M. Lu, and R. M. Osgood, Opt. Lett. 38, 1854 (2013).

11. A. M. J. Koonen, H. Chen, H. V. Boom, and O. Raz, IEEE Photon. Technol. Lett. 24, 1961 (2012).

12. L. H. Gabrielli, D. Liu, S. G. Johnson, and M. Lipson, Nat. Commun. 3, 1217 (2012).

13. L. H. Frandsen, Y. Elesin, L. F. Frellsen, M. Mitrovic, Y. Ding, O. Sigmund, and K. Yvind, Opt. Express 22, 8525 (2014).

14. B. A. Dorin and W. N. Ye, Opt. Express 22, 4547 (2014).

15. Y. Atsumi, J.-H. Kang, Y. Hayashi, J. Suzuki, N. Nishiyama, and S. Arai, Jpn. J. Appl. Phys. 53, 078002 (2014).

16. D. Dai, J. Wang, and Y. Shi, Opt. Lett. 38, 1422 (2013).

17. X. Guan, P. Chen, S. Chen, P. Xu, Y. Shi, and D. Dai, Opt. Lett. 39, 4514 (2014)

18. B. Tatian, Appl. Opt. 23, 4477 (1984).

19. Y. Ding, H. Ou, and C. Peucheret, Opt. Lett. 38, 2732 (2013).

20. Y. Ding, J. Xu, F. D. Ros, H. Ou, and C. Peucheret, Opt. Express 21, 10376 (2013). 